\title{
The orthopaedic community does not oppose the non-surgical treatment of degenerative meniscal lesions
}

\author{
Philippe Beaufils $^{1} \cdot$ Romain Seil ${ }^{2} \cdot$ Roland Becker $^{3} \cdot$ Jon Karlsson $^{4} \cdot$ Jacques Menetrey $^{5}$
}

Received: 6 December 2020 / Accepted: 9 December 2020 / Published online: 3 February 2021

(c) European Society of Sports Traumatology, Knee Surgery, Arthroscopy (ESSKA) 2021

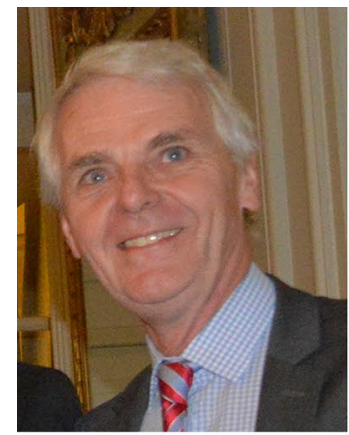

Philippe Beaufils

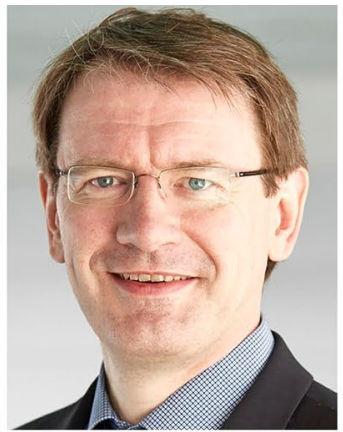

Romain Seil

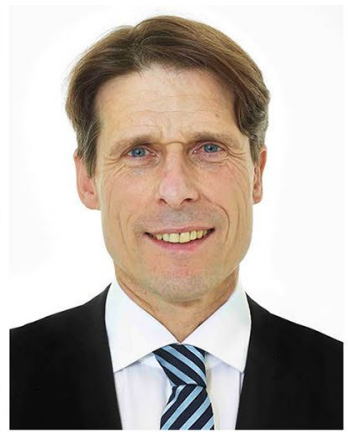

Roland Becker

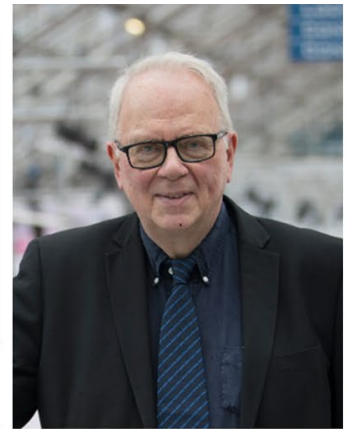

Jon Karlsson

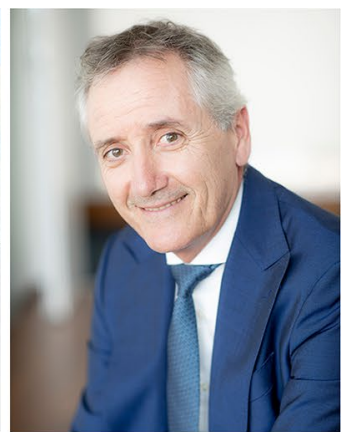

Jacques Menetrey
In its September 2020 issue, the British Journal of Sports Medicine published an editorial "When taking a step back is a veritable leap forward. Reversing decades of arthroscopy for managing joint pain: five reasons that could explain declining rates of common arthroscopic surgeries" written by Ardern and co-workers [1]. In this editorial, which focused on "meniscal" pain in the middle-aged patient (in fact, degenerative meniscal lesions), the authors point out

Philippe Beaufils

drphilippebeaufils@gmail.com

1 ESSKA Consensus Projects Advisor, ESSKA Office Centre Médical, Fondation Norbert Metz, 76, rue d'Eich, L-1460 Luxembourg, Luxembourg

2 ESSKA Past President, ESSKA Office Centre Médical, Fondation Norbert Metz, 76, rue d'Eich, L-1460 Luxembourg, Luxembourg

3 ESSKA Vice President, ESSKA Office Centre Médical, Fondation Norbert Metz, 76, rue d'Eich, L-1460 Luxembourg, Luxembourg

4 Department of Orthopaedics, Sahlgrenska Academy, Sahlgrenska University Hospital, Gothenburg University, Gothenburg, Sweden

5 ESSKA President, ESSKA Office Centre Médical, Fondation Norbert Metz, 76, rue d'Eich, L-1460 Luxembourg, Luxembourg the pre-eminence of non-surgical treatment and they correctly observe the decreasing rate of meniscal surgery over time. However, at the same time, they assert that orthopaedic surgeons and their representative scientific societies would act as a brake and even a resistance to the generalisation of non-surgical treatment, while still generally promoting arthroscopic partial meniscectomy (APM) in degenerative meniscal lesions (DML). They also call on patients to resist and on official public healthcare systems to publish imposed regulations promoting non-surgical treatment or no treatment at all, even in symptomatic patients.

In this context, two important points need to be stressed.

First, meniscal preservation should be a priority, in both young and middle-aged patients. In these populations, the clinical pictures and types of meniscal injury are manifold. Moreover, we all agree that, on the basis of solid scientific studies, the first-line treatment should be non-surgical in most middle-aged and older patients. A strong message is, however, conveyed by the recommendations resulting from a large, scientifically based consensus project, which was organised by ESSKA in 2013 and published in 2017 and 2019 [2]. The publication in 2017 proposed APM in degenerative meniscal lesions in the event of the failure of initial non-surgical treatment and the persistence of mechanical 
symptoms. Early APM was only recommended in specific, clearly depicted and very carefully selected situations.

Second, the decline of APM in this clinical setting is real but probably not as rapid as might otherwise have been expected. In this debate, it should be remembered that implementing science takes time. Other studies which were also carried out by scientific societies showed a significant decrease in the number of meniscectomies, both in France [8] and in the United States [11], for example, thus confirming the role of scientific societies and particularly of ESSKA in the defence of the concept of meniscal preservation. It was surprising to see that these publications have not been cited by the authors. Moreover, they appeared to prefer to discredit the role of scientific societies. This is unfortunate, because science should be balanced and truthful and citations should be correct and comprehensive. It therefore appears that this editorial is scientifically questionable and should even be regarded as dangerous in its form for the medical world.

Furthermore, it appears to be driven predominantly by the authors' impressions and their personal agenda rather than scientific evidence. Two assertions in particular deserve a well-founded discussion. Do orthopaedic surgeons and their scientific societies resist generalising the non-surgical treatment of degenerative meniscal lesions? Do we or the authors of this editorial really know anything about this? A quick literature review makes it possible to affirm the opposite. Several editorials have been published since 2015 in KSSTA, strongly underlining the importance of the meniscal preservation concept $[3,12,13,16]$. ESSKA's investigation - and the subsequent Consensus Statement-involved 21 countries [1] and has been disseminated, in their mother tongues, to orthopaedic surgeons across Europe (English, Italian, French, Spanish, German, among others). It was preceded by recommendations from the French orthopaedic community [4] and was followed by new UK guidelines [18]. As a result, there have been changes in British regulations (which proves just how useful a scientific society can be, as a driver of efficient clinical best practice). By contrast, the editorial by Ardern and co-workers was limited to a few countries and a small number of references. Insufficient citations are not helpful to scientific progress. Likewise, limiting the focus of the discussion to a restricted geographical area does not reflect the diversity of European cultures.

Regarding the slower-than-expected decline in APM, it is appropriate to wonder in retrospect why there is a gap between the "data of science" and the reality of everyday life. ESSKA has just launched a European study on the subject. It is on this basis that the BJSM editorial is incorrect, ineffective, and even dangerous. Considering that the solution lies in stigmatising the orthopaedic community and calling on patients to resist and coerce them through imposed regulations, there is clearly a lack of knowledge of day-today practice which risks creating unnecessary resistance.
This type of conflict has already been experienced in the last decade through various publications $[9,19]$, thereby leading to aggressive, yet useless reactions and discussions $[6,10]$. It was to avoid this type of behaviour that ESSKA initiated the consensus process. Today, rekindling polemics and launching anathemas is useless. Questioning the reasons for the gap between recommendations and everyday practice should be preferred and common solutions integrating all stakeholders (healthcare professionals, patients, civil society, and regulatory bodies) should be identified. There are many reasons for this gap: the acceptance of the conclusions of the cited RCTs by surgeons, the behaviour of patients and more generally of civil society, or the weight of medico-economic constraints and regulations. In this context, we are in agreement with the pressure groups developed in the BJSM editorial; the surgeon, the patient, and the regulatory system. However, we differ fundamentally on the proposed solutions.

Why do we disagree?

First, there is the argument of time. It must be accepted that the dissemination of information takes time, often decades. The concept of meniscal repair is a pre-eminent example of this slow evolution [5, 17].

Second, there is the acceptability of RCTs. However, solid they may be, they are not always easily adopted in daily clinical practice. This is a well-known and well-studied mechanism. Any RCT has its weaknesses and limitations. This should be recognised, particularly in joint surgery, where clinical symptoms often vary and may not be directly related to the structural damage [13]. Selection bias is one of the main arguments. Who can say with certainty that the profile of patients who have agreed to participate in a study in Finland involving sham surgery is representative of the general population in all countries? This raises the question of the external validity of this type of study and represents an additional argument to highlight the value of a well-conducted consensus that balances "scientific data" and "everyday expertise". It may be called "evidence-based medicine" versus "eminence-based medicine", but without the negative connotation of the latter. It is instead the result of an accumulation of clinical data over many decades. Today, this questioning goes far beyond the framework of our discipline and crosses all medical specialities. Some authors [15] point to the need for the so-called "real-life" studies. The ESSKA meniscal consensus, which enlisted more than 80 experts from over 21 European countries, was, in this respect, an exciting experience showing the diversity of cultures and approaches but their ability to find a common path.

Third, like many other professionals, surgeons work within a paradigm, unless or until that paradigm is proven ineffectual (at which point they will adapt and change) [14]. This cannot be otherwise. Moreover, like any human being, they are subject to what is known as confirmation bias or prejudice [7], leading them willingly to accept what 
corresponds to their ideas rather than what is opposed to them. This is a normal, universal behaviour and it is not voluntary. It must be accepted. Anathemas will not solve this, but large-scale educational programmes in which academic structures and scientific societies play an essential role will. ESSKA, as well as its affiliated national societies, is taking a major responsibility in this endeavour.

Fourth, is the patient in a position to form a pressure group in favour of non-surgical treatment? Perhaps one day. We do understand that patient behaviour is fundamentally linked to the specific cultures of each society. However, in many countries, patients who have been suffering from pain for several months and who consult a surgeon are seeking "active" treatment and hardly even think that non-surgical treatment is a way to cure their pain. This is a mistake on their part, of course, but it is one that RCTs ignore. Nor does this mean that we should operate on them. Here, again, consensus, mixing evidence and eminence-based medicine, or "real-life" studies are processes that can facilitate an inclusive education rather than the promotion of opposition between pressure groups.

Finally, there is the diversity in healthcare systems which makes any global approach very difficult. They may be government oriented versus liberal, fee for service, or pathology based. Insurances may be public or private, and both the accessibility and the type of involved healthcare professionals vary widely. This is not the place to go into detail. Using coercion to limit the number of APMs is not the way to go in this diverse landscape. Past experiences in other fields of surgery have revealed their limits. Here, again, consultation, which should be based on solid, shared data, is preferable to confrontation.

In conclusion, meniscal preservation is a major issue and the non-surgical treatment of DML is the first-line treatment. This does not exclude APM in selected cases, in accordance with the international recommendations. The surgical community is not voluntarily opposed to the reduction of APM in this context. It is only through education, exchange, and collaboration, taking account of the cultural differences in each country, and not through confrontation, that a common goal of this kind will be achieved.

\section{Compliance with ethical standards}

Conflict of interest PB: none.

Funding None.

Ethical approval I did not find any ethical approval statement for editorials in KSSTA.

\section{References}

1. Ardern CL, Paatela T, Mattila V et al (2020) When taking a step back is a veritable leap forward. Reversing decades of arthroscopy for managing joint pain: five reasons that could explain declining rates of common arthroscopic surgeries. Br J Sports Med 54:1311-1313

2. Beaufils P, Becker R, Kopf S, Englund M, Verdonk R, Ollivier M, Seil R (2017) Surgical management of degenerative meniscus lesions: the 2016 ESSKA meniscus consensus. Knee Surg Sports Traumatol Arthrosc 25(2):335-346

3. Beaufils P, Becker R, Verdonk R, Aagaard H, Karlsson J (2015) Focusing on results after meniscus surgery. Knee Surg Sports Traumatol Arthrosc 23(1):3-7

4. Beaufils P, Hulet C, Dhénain M, Nizard R, Nourissat G, Pujol $\mathrm{N}$ (2009) Clinical practice guidelines for the management of meniscal lesions and isolated lesions of the anterior cruciate ligament of the knee in adults. Orthop Traumatol Surg Res 95(6):437-442

5. Becker R, Kopf S, Seil R, Hirschmann MT, Beaufils P, Karlsson J (2020) From meniscal resection to meniscal repair: a journey of the last decade. Knee Surg Sports Traumatol Arthrosc 28(11):3401-3404

6. Bollen SR (2015) Is arthroscopy of the knee completely useless? Meta-analysis-a reviewer's nightmare. Bone Jt J 97-B(12):1591-1592

7. Elston DM (2020) Confirmation bias in medical decision-making. J Am Acad Dermatol 82(3):572

8. Jacquet C, Pujol N, Pauly V, Beaufils P, Ollivier M (2019) Analysis of the trends in arthroscopic meniscectomy and meniscus repair procedures in France from 2005 to 2017. Orthop Traumatol Surg Res 105(4):677-682

9. Lohmander LS, Thorlund JB, Roos EM (2016) Routine knee arthroscopic surgery for the painful knee in middle-aged and old patients-time to abandon ship. Acta Orthop 87(1):2-4

10. Lubowitz JH, Provencher MT, Rossi MJ (2014) Could the new england journal of medicine be biased against arthroscopic knee surgery? Part 2. Arthroscopy 30(6):654-655

11. Parker BR, Hurwitz S, Spang J, Creighton R, Kamath G (2016) Surgical trends in the treatment of meniscal tears: analysis of data from the American board of orthopaedic surgery certification examination database. Am J Sports Med 44(7):1717-1723

12. Pujol N, Beaufils P (2019) Save the meniscus again! Knee Surg Sports Traumatol Arthrosc 27(2):341-342

13. Reeves M. EPI-546: Fundamentals of epidemiology and biostatistics. Course Notes: Lecture 7 The RCT. https://learn.chm. msu.edu/epi/Coursepack/EPI546_Lecture_7_course_notes.pdf. Accessed 12 Nov 2020

14. Reider B (2015) Editorial: To Cut ... or Not? Am J Sports Med 43(10):2365-2367

15. Saturni S, Bellini F, Braido F, Paggiaro P, Sanduzzi A, Scichilone N, Santus PA, Morandi L, Papi A (2014) Randomized Controlled Trials and real life studies. Approaches and methodologies: a clinical point of view. Pulm Pharmacol Ther 27(2):129-138

16. Seil R, Karlsson J, Beaufils P, Becker R, Kopf S, Ollivier M, Denti M (2017) The difficult balance between scientific evidence and clinical practice: the 2016 ESSKA meniscus consensus on the surgical management of degenerative meniscus lesions. Knee Surg Sports Traumatol Arthrosc 25(2):333-334

17. Seil R, Becker R (2016) Time for a paradigm change in meniscal repair: save the meniscus! Knee Surg Sports Traumatol Arthrosc 24(5):1421-1423 
18. Siemieniuk RAC, Harris IA, Agoritsas T et al (2018) Arthroscopic surgery for degenerative knee arthritis and meniscal tears: a clinical practice guideline. Br J Sports Med 52:313

19. Thorlund JB, Juhl CB, Roos EM, Lohmander LS (2015) Arthroscopic surgery for degenerative knee: systematic review and metaanalysis of benefits and harms. Br Med J 16(350):h2747
Publisher's Note Springer Nature remains neutral with regard to jurisdictional claims in published maps and institutional affiliations. 